\title{
Bullying y factores de riesgo para la salud en estudiantes de secundaria
}

\author{
Inmaculada Méndez y Fuensanta Cerezo \\ Universidad de Murcia (España)
}

\begin{abstract}
La convivencia entre escolares se está convirtiendo en un grave problema en nuestras aulas. Los docentes tienen serios problemas para hacer frente a conductas perturbadoras entre los estudiantes (agresiones verbales y físicas, consumo de drogas, etc.). Por otra parte, los estudios apuntan que el consumo de drogas forma parte de la vida de muchos adolescentes, considerando que estamos ante una "normalización" de su consumo. El objetivo de nuestra investigación es indagar las posibles asociaciones entre los implicados en bullying y la realización de determinadas conductas de riesgo para la salud (por ejemplo, consumo de sustancias adictivas). Sobre una muestra de 886 estudiantes de Enseñanza Secundaria Obligatoria, se administraron los instrumentos de evaluación siguientes: el Test BULL-S y una encuesta elaborada ah hoc sobre conductas de riesgo para la salud. Los resultados indican que el $20.8 \%$ están implicados en bullying en sus distintos roles. Entre las variables seleccionadas, los agresores destacan en: consumo de tabaco y de alcohol así como en otras conductas que implican un alto riesgo para la salud.
\end{abstract}

Palabras clave: Adolescencia, acoso escolar, drogas, problema social.

Bullying and risk factors for health among students of secondary education. Coexistence among students has grown as a deep problem in our classrooms. Teachers have to face disrupting attitudes (verbal and physical aggressions, drug-taking, etc). Moreover, studies suggest that drug use is part of the lives of many adolescents, considering that this is a "normalization" of consumption. The aim of our investigation is researching into the possible associations between those students who are involved in bullying and determined ways of behaving that imply health risks (drug-taking, etc.) Upon a sample taken from 886 students of Compulsory Secondary Education, the following evaluation instruments were used: the Test BULL-S and a survey - taken $a d$ hoc- about health risks. The results of this study show that a $20.8 \%$ of the students are involved in bullying, playing different roles. Among the selected variables, the bullies are more inclined to consuming tobacco and alcohol, as well as showing other types of behaviour that imply a high risk for their health.

Key words: Adolescence, school bullying, drugs, social problem.

Correspondencia: Inmaculada Méndez. Universidad de Murcia. Facultad de Psicología. Departamento de Psicología Evolutiva y de la Educación. - Espinardo- C.P. 30.100 Murcia (España). E-mail: minmaculada.mendez@um.es 
El Estudio Internacional sobre Enseñanza y Aprendizaje, TALIS (Teaching and Learning Internacional Survey), sitúa a España por encima de la media internacional en problemas de clima escolar. Los problemas de convivencia entre escolares contribuyen a crear y a la vez son emergentes de climas sociales negativos, en los que no es difícil encontrar fenómenos de violencia entre compañeros (Ortega, 2000). A ésta conducta agresiva que se manifiesta entre escolares se la conoce, internacionalmente, con el nombre de "bullying" (Olweus, 1998). El fenómeno bullying se define como una forma de maltrato, normalmente intencionado, perjudicial y persistente de un estudiante o grupo de estudiantes, hacia otro compañero al que convierte en su víctima habitual. El bullying se expresa tanto con agresiones físicas como verbales y como exclusión. Se presenta como un fenómeno grupal, desde el momento en que precisa del soporte del grupo y por tanto, debe situarse en la trama de relaciones que en el mismo se genera. Por tanto, para su estudio, se hace imprescindible incluir el análisis de las relaciones socioafectivas, las condiciones ambientales donde se producen (variables situacionales) y la percepción de gravedad que los escolares le atribuyen (Cerezo, 2008; 2009a).

Por otro lado, la adolescencia es considerada como una etapa de especial riesgo para la adquisición de determinadas conductas perjudiciales para la salud, tales como el consumo de determinadas sustancias adictivas y la realización de determinadas conductas problemáticas. El reciente informe de la Delegación del Gobierno para el Plan Nacional sobre Drogas -DGPNSD-(2009) nos informa del preocupante número de estudiantes que realizan actividades de riesgo para su salud como por ejemplo, la alta frecuencia de emborracharse, el policonsumo, etc.

Se han llevado a cabo investigaciones que demuestran que se está produciendo un notable incremento tanto en el número de adolescentes que se inician en el consumo de drogas como en el de sujetos que presentan actividades delictivas (Otero, 2001). Herrero (2003), destaca que es difícil establecer una relación de causa-efecto entre consumos de alcohol y drogas y determinadas conductas inadaptadas o de riesgo, pero aún así resulta evidente la relación existente entre ellas. Investigaciones realizadas concretamente con la problemática bullying ponen de manifiesto que los acosadores coinciden con ser los mayores consumidores de alcohol y otras sustancias, seguidos de los víctimas-provocadores (Kaltiala-Heino, Rimpelä, Rantanen y Rimpelä, 2000; Elzo, 2005; Serrano e Iborra, 2005).

Teniendo en cuenta las investigaciones previas, consideramos oportuno llevar a cabo un estudio piloto que nos acercase al problema de la violencia entre escolares y su asociación con el consumo de sustancias adictivas en dichos escolares. En ese estudio obtuvimos una asociación entre los implicados en la problemática bullying y la realización de determinadas conductas de riesgo, especialmente significativas entre los agresores (Cerezo y Méndez, 2009; Méndez, 2009). Los resultados de este estudio piloto sentaron las bases de la investigación que presentamos. La finalidad de nuestra 
investigación es la de poder indagar, en estudiantes de Educación Secundaria Obligatoria, la posible asociación entre la realización de ciertas conductas o factores de riesgo para la salud (consumo de sustancias adictivas, policonsumo, etc.) y los problemas de convivencia entre dichos estudiantes (perfiles asociados en la problemática bullying).

\section{METODO}

\section{Participantes}

La muestra estuvo compuesta por 886 estudiantes de Enseñanza Secundaria Obligatoria ( $41.8 \%$ de primer ciclo y $58.2 \%$ de segundo ciclo) en centros de enseñanza públicos y privados de la provincia de Murcia. Siendo la siguiente distribución por curso: $27.8 \%$ de $1^{\circ} ; 14 \%$ de $2^{\circ} ; 24.9 \%$ de $3^{\circ}$ y $33.3 \%$ de $4^{\circ}$ de ESO. Con un rango de edad entre 11 y 18 años, edad media 14.52 y desviación típica 1.447. Este rango se justifica porque el $40.5 \%$ de los estudiantes habían repetido curso alguna vez; y el $10.5 \%$ había repetido dos cursos o más. El $47.7 \%$ eran varones y el $52.3 \%$ eran mujeres. Asimismo, se obtuvo que un $18.3 \%$ de los estudiantes eran de procedencia extranjera.

\section{Instrumentos}

Se seleccionaron dos instrumentos de evaluación anónimos y de aplicación colectiva. El “Test Bull-S” (Cerezo, 2000/2002) para la recogida de datos sobre las variables implicadas en la problemática bullying, a través del análisis de la estructura interna del aula desde la perspectiva de los estudiantes. Está compuesto por 15 ítems, que evalúan las siguientes dimensiones de información: el estudio de la estructura interna del aula, mediante criterios de aceptación- rechazo (formada por 4 ítems de elección directa); la dinámica bullying a través de 6 ítems, evaluando aspectos referidos a las características asociadas a los sujetos implicados en la problemática bullying y una tercera categoría en formato Likert que mide los aspectos situacionales. Los análisis estadísticos constatan la validez y fiabilidad de dicha prueba en unos límites muy aceptables. Usando las variables relativas a agresión y victimización, el alfa de Cronbach para $n=322$, fue de .73 y puntuó de manera similar en diferentes grupos de edad, sexo y aulas (valores situados entre .69 y .75). Este valor aumento cuando fueron separados los ítems relativos a las conductas de victimización (.83) y para los relativos a la agresión (.82) (Cerezo, 2006, 2009b).

Para la recogida de datos sobre el consumo de sustancias adictivas y la realización de conductas problemáticas para la salud se utilizó una encuesta elaborada $a d$ hoc basándose en la "Encuesta estatal sobre uso de drogas en estudiantes de Enseñanzas Secundarias (ESTUDES)”, promovida por la Delegación del Gobierno para el Plan Nacional sobre Drogas (2008), que permite conocer la situación y las tendencias del 
consumo de drogas entre los estudiantes (Cerezo, Méndez y Rabadán 2009). Está formada por 82 ítems (preguntas dicotómicas y politómicas) agrupados en cinco bloques: características sociodemográficas, consumo de drogas, problemas que el estudiante ha podido tener en los últimos 12 meses, hábitos deportivos y salud y percepción de la convivencia escolar.

\section{Procedimiento}

En un primer momento se llevó a cabo la autorización de la Consejería de Educación, Formación y Empleo de la Región de Murcia. Tras la selección de los centros de enseñanza secundaria, se realizó una entrevista con los directores y/u orientadores de los centros educativos para exponer los objetivos de la investigación, describir los instrumentos de evaluación, solicitar su permiso y promover su colaboración. Posteriormente se informó al Consejo Escolar y se obtuvo la autorización de los padres, madres o representantes legales de los estudiantes.

En las sesiones de aplicación (dos sesiones de 50 minutos) de los instrumentos de evaluación, se informó a los estudiantes del carácter voluntario, anónimo y confidencial del estudio así como de los objetivos del mismo. Las hojas de respuesta fueron codificadas e introducidas en una base de datos para su tratamiento con el paquete estadístico SPSS (versión 15.0). El diseño utilizado fue transversal, de tipo descriptivo; utilizando técnicas estadísticas inferenciales, tales como análisis de frecuencias y tablas de contingencia a través de la prueba Chi-cuadrado, para buscar asociaciones significativas entre las variables de estudio.

\section{RESULTADOS}

\section{Problemática bullying}

En cuanto a la problemática bullying, los datos del análisis estadístico del Test Bull-S, proporcionan información de los estudiantes que destacan en al menos un 25\% en cada uno de los perfiles asociados a la dinámica bullying. En el gráfico 1 se puede observar la distribución de la muestra: aparece cerca del $80 \%$ de neutros, una proporción igualada de bullies y de víctimas, siendo menor la de víctimas-provocadores $(1.7 \%)$.

Gráfico 1. Incidencia de problemática bullying en la muestra

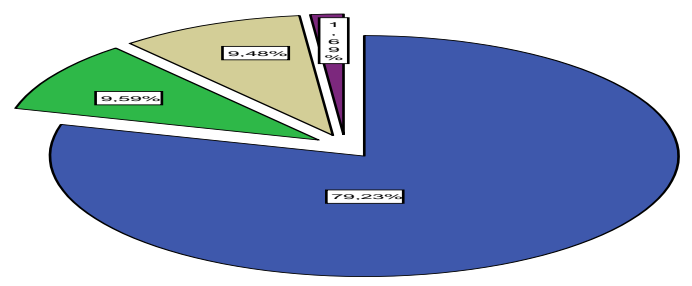


La tabla 1 presenta la distribución de la muestra por roles asignados según el sexo. Puede apreciarse una mayoría del sexo masculino entre los implicados en los distintos roles de la problemática bullying, resultando estadísticamente significativo.

Tabla 1. Distribución de la muestra en función del sexo

\begin{tabular}{|c|c|c|c|c|c|c|c|}
\hline \multicolumn{2}{|c|}{$\begin{array}{l}\text { DISTRIBUCIÓN } \\
\text { MUESTRA/ } \\
\text { SEXO }\end{array}$} & 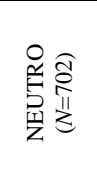 & 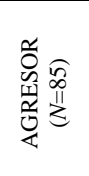 & 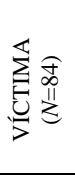 & 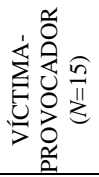 & 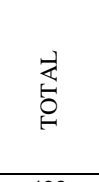 & $\begin{array}{c}\text { VALOR } \\
\text { PRUEBA } \\
\text { CHI-CUADRADO }\end{array}$ \\
\hline \multirow{2}{*}{ SEXO } & Hombre & $\begin{array}{c}284 \\
(40.5 \%) \\
\end{array}$ & $\begin{array}{c}65 \\
(76.5 \%) \\
\end{array}$ & $\begin{array}{c}63 \\
(75 \%) \\
\end{array}$ & $\begin{array}{c}11 \\
(73.3 \%) \\
\end{array}$ & $\begin{array}{c}423 \\
(47.7 \%) \\
\end{array}$ & \multirow{2}{*}{$\begin{array}{c}\text { Chi-cuadrado(3) }=72.009, \\
p=.000\end{array}$} \\
\hline & Mujer & $\begin{array}{c}418 \\
(59.5 \%) \\
\end{array}$ & $\begin{array}{c}20 \\
(23.5 \%)\end{array}$ & $\begin{array}{c}21 \\
(25 \%) \\
\end{array}$ & $\begin{array}{c}4 \\
(26.7 \%) \\
\end{array}$ & $\begin{array}{c}463 \\
(52.3 \%) \\
\end{array}$ & \\
\hline
\end{tabular}

En cuanto a la procedencia de los estudiantes, según los roles asignados en la problemática bullying, se obtuvo que eran extranjeros los siguientes: neutro (14.2\%), agresor (1.6\%), víctima (2\%) y víctima-provocador $(.5 \%)$.

El la tabla 2 se puede apreciar la distribución de la muestra atendiendo al factor repetición, es decir, aquellos estudiantes que han repetido un curso o más, frente a los que no han repetido curso, en función de los roles asociados a la problemática bullying.

Tabla 2. Distribución de la muestra por factor repetición

\begin{tabular}{|c|c|c|c|c|c|c|c|}
\hline $\begin{array}{r}\text { DISTRIBUC } \\
\text { REF }\end{array}$ & $\begin{array}{l}\text { SN MUESTRA/ } \\
\text { [ICIÓN }\end{array}$ & 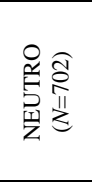 & 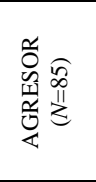 & 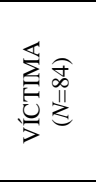 & 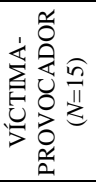 & $\begin{array}{l}\frac{1}{6} \\
\circ \\
\end{array}$ & $\begin{array}{c}\text { VALOR } \\
\text { PRUEBA } \\
\text { CHI- } \\
\text { CUADRADO }\end{array}$ \\
\hline \multirow{3}{*}{ REPETICIÓN } & Un curso & $\begin{array}{c}199 \\
(28.3 \%)\end{array}$ & $\begin{array}{c}35 \\
(41.2 \%)\end{array}$ & $\begin{array}{c}28 \\
(33.3 \%)\end{array}$ & $\begin{array}{c}4 \\
(26.7 \%)\end{array}$ & $\begin{array}{c}266 \\
(30 \%)\end{array}$ & \multirow{3}{*}{$\begin{array}{l}\text { Chi-cuadrado(9) } \\
=24.129, p=.004\end{array}$} \\
\hline & Dos o más cursos & $\begin{array}{c}68 \\
(9.7 \%)\end{array}$ & $\begin{array}{c}18 \\
(21.2 \%)\end{array}$ & $\begin{array}{c}5 \\
(6 \%)\end{array}$ & $\begin{array}{c}2 \\
(13.3 \%)\end{array}$ & $\begin{array}{c}93 \\
(10.5 \%)\end{array}$ & \\
\hline & Ninguno & $\begin{array}{c}435 \\
(62 \%)\end{array}$ & $\begin{array}{c}32 \\
(37.6 \%)\end{array}$ & $\begin{array}{c}51 \\
(60.7 \%)\end{array}$ & $\begin{array}{c}9 \\
(60 \%)\end{array}$ & $\begin{array}{c}527 \\
(59.5 \%) \\
\end{array}$ & \\
\hline
\end{tabular}

La distribución por edad de los estudiantes atendiendo al rol asignado en la problemática bullying, se presenta del siguiente modo: neutros 14.52 (desviación típica de 1.46); agresores 14.69 años (desviación típica de 1.32); víctimas 14.30 años (desviación típica de 1.55) y víctimas-provocadores 14.67 años (desviación típica de $.98)$.

En la tabla 3 se pueden apreciar los aspectos situacionales de la problemática bullying, los cuales nos proporcionan información de elementos concretos de las situaciones de agresión entre escolares, explicitando: la forma que adoptan, dónde suelen tener lugar, con qué frecuencia ocurren y el nivel de gravedad que le atribuyen los estudiantes. 
MENDEZ y CEREZO. Bullying y factores de riesgo para la salud

Tabla 3. Aspectos situacionales de la problemática bullying

\begin{tabular}{|c|c|c|}
\hline \multicolumn{2}{|c|}{$\begin{array}{l}\text { VARIABLES SITUACIONALES } \\
\text { PROBLEMÁTICA BULLYING }\end{array}$} & PORCENTAJE \\
\hline \multirow{4}{*}{ Tipo de agresiones } & Insultos y amenazas & $53.2 \%$ \\
\hline & Rechazo & $35.8 \%$ \\
\hline & Maltrato físico & $7.9 \%$ \\
\hline & Otras formas & $3.1 \%$ \\
\hline \multirow{4}{*}{ Lugar de las agresiones } & Aula & $46.4 \%$ \\
\hline & Patio & $34.2 \%$ \\
\hline & Pasillos & $12.8 \%$ \\
\hline & Otros sitios & $6.6 \%$ \\
\hline \multirow{4}{*}{ Frecuencia de las agresiones } & Todos los días & $8.9 \%$ \\
\hline & Una o dos veces por semana & $46 \%$ \\
\hline & Rara vez & $28.4 \%$ \\
\hline & Nunca & $16.6 \%$ \\
\hline \multirow{4}{*}{ Gravedad de las agresiones } & Poco o nada & $32.4 \%$ \\
\hline & Regular & $36.8 \%$ \\
\hline & Bastante & $19.9 \%$ \\
\hline & Mucho & $10.9 \%$ \\
\hline \multirow{4}{*}{ Seguridad en el centro educativo } & Poco o nada & $4.8 \%$ \\
\hline & Regular & $14.9 \%$ \\
\hline & Bastante & $40.1 \%$ \\
\hline & Mucho & $40.2 \%$ \\
\hline
\end{tabular}

Tabla 4. Distribución de la muestra en consumo de sustancias adictivas

\begin{tabular}{|c|c|c|c|c|c|}
\hline $\begin{array}{l}\text { DISTRIBUCIÓN MUESTRA/ } \\
\text { CONSUMO } \\
\text { SUSTANCIAS ADICTIVAS }\end{array}$ & 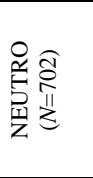 & 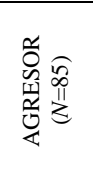 & 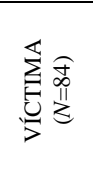 & 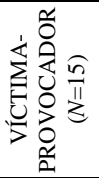 & $\begin{array}{l}\text { VALOR PRUEBA } \\
\text { CHI-CUADRADO }\end{array}$ \\
\hline $\begin{array}{l}\text { Fuma cigarrillos de tabaco } \\
\text { diariamente }\end{array}$ & $\begin{array}{c}91 \\
(13 \%) \\
\end{array}$ & $\begin{array}{c}20 \\
(23.5 \%)\end{array}$ & $\begin{array}{c}1 \\
(1.2 \%) \\
\end{array}$ & $\begin{array}{c}3 \\
(20 \%) \\
\end{array}$ & $\begin{array}{c}\text { Chi-cuadrado }(6)=21.311, \\
p=.002\end{array}$ \\
\hline $\begin{array}{l}\text { Toma bebidas alcohólicas todas las } \\
\text { semanas }\end{array}$ & $\begin{array}{c}102 \\
(14.5 \%)\end{array}$ & $\begin{array}{c}24 \\
(28.2 \%)\end{array}$ & $\begin{array}{c}6 \\
(7.1 \%) \\
\end{array}$ & $\begin{array}{c}4 \\
(26.7 \%) \\
\end{array}$ & $\begin{array}{c}\text { Chi-cuadrado }(6)=20.766, \\
p=.002\end{array}$ \\
\hline Se ha emborrachado & $\begin{array}{c}232 \\
(33 \%) \\
\end{array}$ & $\begin{array}{c}45 \\
(52.9 \%) \\
\end{array}$ & $\begin{array}{c}9 \\
(10.7 \%) \\
\end{array}$ & $\begin{array}{c}6 \\
(40 \%) \\
\end{array}$ & $\begin{array}{c}\text { Chi-cuadrado }(6)=36.117, \\
p=.000\end{array}$ \\
\hline $\begin{array}{l}\text { Ha consumido tranquilizantes sin } \\
\text { receta médica }\end{array}$ & $\begin{array}{c}57 \\
(8.1 \%)\end{array}$ & $\begin{array}{c}5 \\
(5.9 \%)\end{array}$ & $\begin{array}{c}5 \\
(6 \%)\end{array}$ & $\begin{array}{c}2 \\
(13.3 \%)\end{array}$ & n.s \\
\hline $\begin{array}{l}\text { Ha consumido alcohol y marihuana } \\
\text { en menos de dos horas }\end{array}$ & $\begin{array}{c}148 \\
(20.6 \%)\end{array}$ & $\begin{array}{c}40 \\
(47 \%) \\
\end{array}$ & $\begin{array}{c}11 \\
(10.7 \%)\end{array}$ & $\begin{array}{c}6 \\
(40 \%) \\
\end{array}$ & $\begin{array}{c}\text { Chi-cuadrado }(18)=59.067, \\
p=.000\end{array}$ \\
\hline $\begin{array}{l}\text { Ha consumido alcohol y cocaína en } \\
\text { menos de dos horas }\end{array}$ & $\begin{array}{c}60 \\
(7.9 \%)\end{array}$ & $\begin{array}{c}19 \\
(22.4 \%)\end{array}$ & $\begin{array}{c}11 \\
(10.7 \%)\end{array}$ & $\begin{array}{c}3 \\
(20 \%) \\
\end{array}$ & $\begin{array}{c}\text { Chi-cuadrado }(18)=33.492, \\
p=.015\end{array}$ \\
\hline
\end{tabular}

\section{Problemática bullying y consumo de sustancias adictivas}

Se presentan en la tabla 4 los resultados obtenidos al comparar en la distribución de la muestra, en la prueba Chi-cuadrado, algunas variables de ambos instrumentos de evaluación teniendo en cuenta el rol asignado en la problemática bullying. Asimismo, pueden observarse los valores para las comparaciones que resultaron ser significativas a diferencia de otras. Los valores son expresados en porcentajes en función del total de cada subgrupo que ha contestado positivamente en cada una de ellas. 


\section{Problemática bullying y conductas de riesgo para la salud en los últimos}

\section{2 meses}

En la tabla 5 se muestran los resultados tras comparar, en la prueba Chi-cuadrado, algunas variables de ambos instrumentos de evaluación teniendo en cuenta la distribución de la muestra en función del rol asignado en la problemática bullying. Los valores son expresados en porcentajes en función del total de cada subgrupo que ha contestado positivamente en cada una de ellas.

Tabla 5. Distribución de la muestra en conductas de riesgo para la salud

\begin{tabular}{|c|c|c|c|c|c|}
\hline $\begin{array}{l}\text { DISTRIBUCÓN MUESTRA/ } \\
\text { CONDUCTAS DE RIESGO } \\
\text { EN LOS ÚLTIMOS } 12 \text { MESES }\end{array}$ & 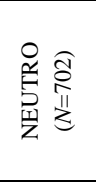 & 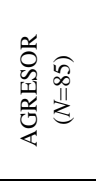 & 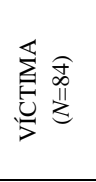 & 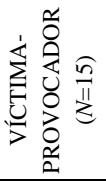 & $\begin{array}{l}\text { VALOR PRUEBA } \\
\text { CHI-CUADRADO }\end{array}$ \\
\hline $\begin{array}{l}\text { Ha conducido bajo los efectos del } \\
\text { alcohol }\end{array}$ & $\begin{array}{c}55 \\
(7.2 \%)\end{array}$ & $\begin{array}{c}19 \\
(22.4 \%)\end{array}$ & $\begin{array}{c}11 \\
(11.9 \%)\end{array}$ & $\begin{array}{c}0 \\
(.0 \%)\end{array}$ & $\begin{array}{c}\text { Chi-cuadrado(18)=41.247, } \\
p=.001\end{array}$ \\
\hline $\begin{array}{l}\text { Había consumido alcohol antes de } \\
\text { participar en una pelea }\end{array}$ & $\begin{array}{c}36 \\
(5.1 \%) \\
\end{array}$ & $\begin{array}{c}10 \\
(11.8 \%) \\
\end{array}$ & $\begin{array}{c}1 \\
(1.2 \%) \\
\end{array}$ & $\begin{array}{c}1 \\
(6.7 \%) \\
\end{array}$ & $\begin{array}{c}\text { Chi-cuadrado(6) }=30.059, \\
p=.000\end{array}$ \\
\hline $\begin{array}{l}\text { Había consumido marihuana antes } \\
\text { de participar en una pelea }\end{array}$ & $\begin{array}{c}13 \\
(1.9 \%)\end{array}$ & $\begin{array}{c}9 \\
(10.6 \%)\end{array}$ & $\begin{array}{c}1 \\
(1.2 \%)\end{array}$ & $\begin{array}{c}0 \\
(.0 \%)\end{array}$ & $\begin{array}{c}\text { Chi-cuadrado }(6)=40.870, \\
p=.000\end{array}$ \\
\hline $\begin{array}{l}\text { Ha sido detenido por la policía o } \\
\text { la guardia civil }\end{array}$ & $\begin{array}{c}39 \\
(5.6 \%) \\
\end{array}$ & $\begin{array}{c}14 \\
(16.5 \%) \\
\end{array}$ & $\begin{array}{c}2 \\
(2.4 \%) \\
\end{array}$ & $\begin{array}{c}2 \\
(13.3 \%) \\
\end{array}$ & $\begin{array}{c}\text { Chi-cuadrado(6)=22.583, } \\
p=.001\end{array}$ \\
\hline $\begin{array}{l}\text { Había consumido marihuana antes } \\
\text { de ser detenido por las fuerzas del } \\
\text { orden público }\end{array}$ & $\begin{array}{c}7 \\
(1 \%)\end{array}$ & $\begin{array}{c}5 \\
(5.9 \%)\end{array}$ & $\begin{array}{c}2 \\
(2.4 \%)\end{array}$ & $\begin{array}{c}0 \\
(.0 \%)\end{array}$ & $\begin{array}{c}\text { Chi-cuadrado(6) }=20.610, \\
\quad p=.002\end{array}$ \\
\hline $\begin{array}{l}\text { Ha sido expulsado del centro } \\
\text { educativo }\end{array}$ & $\begin{array}{c}26 \\
(3.7 \%) \\
\end{array}$ & $\begin{array}{c}20 \\
(23.5 \%) \\
\end{array}$ & $\begin{array}{c}3 \\
(3.6 \%) \\
\end{array}$ & $\begin{array}{c}5 \\
(33.3 \%) \\
\end{array}$ & $\begin{array}{c}\text { Chi-cuadrado(6)=73.257, } \\
p=.000\end{array}$ \\
\hline $\begin{array}{l}\text { Considera que realiza actividades } \\
\text { que ponen en riesgo su salud }\end{array}$ & $\begin{array}{c}108 \\
(15.4 \%)\end{array}$ & $\begin{array}{c}27 \\
(31.8 \%) \\
\end{array}$ & $\begin{array}{c}13 \\
(15.5 \%) \\
\end{array}$ & $\begin{array}{c}3 \\
(20 \%) \\
\end{array}$ & $\begin{array}{c}\text { Chi-cuadrado }(6)=15.980, \\
p=.014\end{array}$ \\
\hline
\end{tabular}

\section{DISCUSION}

Los resultados obtenidos en cuanto a la problemática bullying nos muestran que la tasa de prevalencia de estudiantes agresores es ligeramente superior a la de víctimas, siendo la de víctimas- provocadores la tasa menor. Pues éstos últimos son víctimas que de modo activo se enfrentan a la situación de abuso que están padeciendo por parte de otros compañeros (Díaz-Aguado, 2005). Es de destacar que aparecen más hombres que mujeres implicados en dicha problemática incluso entre los víctimas provocadores. Este dato concuerda con lo mencionado por Cerezo (2001), donde afirmaba que el bullying entre las chicas es menos visible y más rebuscado. La edad de los estudiantes implicados en la problemática bullying abarca el tramo de los 11 a los 18 años de edad, por lo que se observa que el fenómeno bullying se va generalizando a todas las edades escolares, lo que Cerezo (2009a) denomina una "ampliación hacia los extremos". El análisis de la edad media nos informa de que es superior la de los agresores, seguida de los víctimas-provocadores y finalmente de las víctimas; siendo por 
lo tanto la edad media de estos últimos más acorde con la edad media del grupo, datos que coinciden con las investigaciones de Cerezo (1998). Otro dato a considerar es la existencia de más víctimas que bullies de nacionalidad extranjera implicados en la problemática bullying. Así como el hecho de que en todos los roles de los implicados en la problemática bullying aparecieron estudiantes repetidores, siendo superior el número de bullies repetidores que el de víctimas y víctimas-provocadores. Éstos datos coinciden con las conclusiones de algunos estudios (Cerezo 1998; Pelegrín y Garcés de Los Fayos, 2008) en los cuales los agresores están más inadaptados escolarmente, por lo que es posible que no sigan el ritmo de aprendizaje del grupo; hayan repetido curso alguna vez e incluso tienen un mayor riesgo de desarrollar conducta antisocial.

El análisis de los aspectos situacionales de la problemática bullying muestran que: lo más frecuente fueron los insultos y amenazas seguido del rechazo, que ocurrían principalmente en el aula o en el patio con una frecuencia de "una o dos veces por semana" o "rara vez". En cuanto a la percepción de gravedad de dichas situaciones, se obtuvo que los estudiantes consideraban las situaciones como "poco o nada" -"regular" de graves e incluso afirmaban sentirse "bastante" y "muy seguros" en el centro educativo. En general, los datos obtenidos coinciden con las conclusiones aportadas por Cerezo (2009a, 2009b) a lo largo de sus investigaciones con el Test Bull-S.

Del análisis de la relación entre los roles asignados en la problemática bullying y las variables relacionadas con el consumo de sustancias adictivas y la realización de conductas o factores de riesgo para la salud, se observa que, en general, los bullies, a diferencia de los víctimas, no solo afirmaron que consumían más cigarrillos de tabaco diariamente y más bebidas alcohólicas todas las semanas, llegando a emborracharse, sino que incluso habían consumido más de una sustancia, es decir, un policonsumo (alcohol y marihuana o alcohol y cocaína) sin que hubiesen pasado al menos dos horas entre el consumo de una y otra. Por último, en cuanto a la realización de conductas de riesgo para la salud en los últimos 12 meses, se encontró que aparecían más bullies, a diferencia de las víctimas y de los víctimas-provocadores, que habían conducido un vehículo de motor bajo los efectos del alcohol. Incluso cuando se les preguntaba si habían participado en alguna pelea o agresión física, se obtuvo que los bullies mayoritariamente habían consumido alcohol o marihuana dos horas antes. Así como, el hecho de que han aparecido más bullies que habían sido expulsados del centro educativo, seguidos de los víctimas-provocadores. Aparece un dato que nos proporciona información sobre las dimensiones que está tomando dicha problemática actualmente, pues aparecen mayoritariamente más bullies que habían sido detenidos por la policía o la guardia civil en los últimos 12 meses; aunque lo sorprendente es que algunos de los detenidos, tanto bullies como víctimas, afirmaron haber consumido marihuana antes de la detención. Un dato que merece especial interés es que entre los estudiantes de Educación Secundaria, es relativamente frecuente el consumo de tranquilizantes sin 
receta médica (7.8\% del total). Éste dato reforzaría las conclusiones del último informe de la DGPNSD (2009) donde se destacaba el aumento tan desmesurado del consumo de tranquilizantes sin receta médica e incluso la normalización que se está produciendo de este consumo por la baja percepción de riesgo que se tiene. Del mismo modo, cuando se les pregunta si consideran que realizan actividades que ponen en riesgo su salud, se ha obtenido que entre todo el alumnado un $82.6 \%$ del total han dado una respuesta afirmativa.

Esta investigación ha sido una extensión de un estudio piloto realizado anteriormente (Cerezo y Méndez, 2009; Méndez, 2009) lo cual permitió, no solo el poder aumentar considerablemente la muestra del estudio, sino poder incluir otras variables que nos aproximan a entender la asociación que puede existir entre determinados factores de riesgo para la salud y la problemática bullying. En ésta investigación, se han tenido en cuenta diversas sustancias adictivas y la realización de conductas de riesgo para la salud relacionadas directamente con el consumo de sustancias adictivas. De los resultados obtenidos, cabe destacar que: los estudiantes agrupados según el rol asignado en la problemática bullying (Neutro, Agresor, Víctima y Víctima-provocador), ofrecen comportamientos de riesgo diferentes, en su gran mayoría estadísticamente significativas, entre los que destaca el agresor como: mayor consumidor de tabaco diariamente, alcohol todas las semanas, incluso emborrachándose, policonsumo en menos de dos horas (alcohol y marihuana o alcohol y marihuana), conducir bajo los efectos del alcohol, estar involucrado en peleas o agresiones estando bajo los efectos del alcohol o de marihuana, ser detenido por la policía o la guardia civil habiendo consumido marihuana, ser expulsado de su centro educativo y sobre todo, considerando él mismo que se encuentra envuelto en conductas o factores de riesgo para su salud.

Los resultados nos han permitido afianzar los datos obtenidos en el estudio piloto y avanzar en el conocimiento de los perfiles de cada uno de los implicados en la problemática bullying, teniendo en cuenta otros factores de riesgo asociados. Este conocimiento facilitará la elaboración de planes de prevención, así como hacernos conscientes del alcance de los riesgos de los adolescentes escolares de Educación Secundaria, de manera que se hace imprescindible implantar estrategias de intervención dirigidas a los implicados y sobre todo la delimitación de responsabilidades no solo de los docentes sino también de la familia y de las instituciones implicadas.

\section{REFERENCIAS}

Cerezo, F. (1998). Conductas agresivas en la edad escolar. Madrid: Pirámide.

Cerezo, F. (2000/2002). Bull-S. Test de Evaluación de la Agresividad entre Escolares. Madrid/ Bizkaia: Albor-Cohs. 
Cerezo, F. (2001). Variables de personalidad asociadas a la dinámica bullying (agresores versus víctimas) en niños y niñas de 10 a 15 años. Anales de Psicología, 17(1), 37-44.

Cerezo, F. (2006). Violencia y victimización entre escolares. El bullying: estrategias de identificación y elementos para la intervención a través del Test Bull-S. Revista Electrónica de Investigación Psicoeducativa, 9(4), 333-352.

Cerezo, F. (2008). Agresores y Víctimas del Bullying. Desigualdades de género en la violencia entre escolares. Revista de Informació Psicológica, 94, 49-59.

Cerezo, F. (2009a) Bullying: análisis de la situación en las aulas españolas. Internacional Journal of Psychology and Psychological Therapy, 9(3), 383-394.

Cerezo, F. (2009b). La violencia en las aulas. Análisis y propuestas de intervención. Madrid: Pirámide.

Cerezo, F. y Méndez, I. (2009). Adolescentes, agresividad y conductas de riesgo de salud: análisis de variables relacionadas. International Journal of Developmental Psychology, 1(1), 217-226.

Cerezo, F., Méndez, I. y Rabadán, R. (2009). Encuesta para Estudiantes de Enseñanzas Secundarias. No publicado.

Delegación del Gobierno para el Plan Nacional sobre Drogas (DGPNSD) (2008). Estudes 2008. Madrid: Ministerio de Sanidad y Política Social.

Delegación del Gobierno para el Plan Nacional sobre Drogas (DGPNSD) (2009). Informe de la Encuesta Estatal sobre Uso de Drogas en Estudiantes de Enseñanzas Secundarias (ESTUDES) 2008. Madrid: Ministerio de Sanidad y Política Social.

Díaz-Aguado, M.J. (2005). La violencia entre iguales en la adolescencia y su prevención desde la escuela. Psicothema, 17(4), 549-558.

Elzo, J. (2005). Los adolescentes de hoy. Consumo de alcohol y drogas y su relación con los comportamientos violentos. Revista de la Asociación proyecto hombre, 54, 8-14.

Herrero, M.N. (2003). Adolescencia, grupo de iguales, consumo de drogas, y otras conductas problemáticas. Revista de Estudios de Juventud. Aspectos psicosociales de la violencia juvenil, 62, 81-91.

Kaltiala-Heino, R., Rimpelä, M., Rantanen, P. y Rimpelä, A. (2000). Bullying at school: an indicator of adolescents at risk for mental disorders. Journal of Adolescence 23, 661-674.

Méndez, I. (2009). Consumo de sustancias adictivas en la problemática bullying en una muestra de estudiantes murcianos de secundaria. Tesina de Licenciatura. Universidad de Murcia. No publicado.

OCDE (2009). Creating Effective Teaching and Learning Environments. First Results from TALIS.

Olweus, D. (1998). Conductas de acoso y amenaza entre escolares. Madrid: Morata.

Otero, J.M. (2001). Consumo de drogas y comportamientos delictivos en la adolescencia. En C. Saldaña (Ed.), Detección y prevención en el aula de los problemas del adolescente (pp. 179-212). Madrid: Pirámide.

Ortega, R. (2000). Educar la convivencia para prevenir la violencia. Madrid: Aprendizaje.

Pelegrín, A. y Garcés de Los Fayos, E.J. (2008). Variables contextuales y personales que inciden en el comportamiento violento del niño. European Journal of Education and Psychology, 1(1), 5-20.

Serrano, A. y Iborra, I. (2005). Violencia entre compañeros en la escuela. Centro Reina Sofía para el estudio de la violencia. Valencia: Serie Documentos. 